\title{
Stability and bifurcation analysis of spin-polarized vertical-cavity surface-emitting lasers
}

\author{
Nianqiang Li, ${ }^{1}$ H. Susanto, ${ }^{2}$ B. R. Cemlyn, ${ }^{1}$ I. D. Henning, ${ }^{1}$ and M. J. Adams ${ }^{1}$ \\ ${ }^{1}$ School of Computer Science and Electronic Engineering, University of Essex, Wivenhoe Park, Colchester CO4 3SQ, United Kingdom \\ ${ }^{2}$ Department of Mathematical Sciences, University of Essex, Wivenhoe Park, Colchester CO4 3SQ, United Kingdom \\ (Received 16 February 2017; revised manuscript received 23 April 2017; published 20 July 2017)
}

\begin{abstract}
A detailed stability and bifurcation analysis of spin-polarized vertical-cavity surface-emitting lasers (VCSELs) is presented. We consider both steady-state and dynamical regimes. In the case of steady-state operation, we carry out a small-signal (asymptotic) stability analysis of the steady-state solutions for a representative set of spin-VCSEL parameters. Compared with full numerical simulation, we show this produces surprisingly accurate results over the whole range of pump ellipticity, and spin-VCSEL bias up to 1.5 times the threshold. We then combine direct numerical integration of the extended spin-flip model and standard continuation technique to examine the underlying dynamics. We find that the spin VCSEL undergoes a period-doubling or quasiperiodic route to chaos as either the pump magnitude or polarization ellipticity is varied. Moreover, we find that different dynamical states can coexist in a finite interval of pump intensity, and observe a hysteresis loop whose width is tunable via the pump polarization. Finally we report a comparison of stability maps in the plane of the pump polarization against pump magnitude produced by categorizing the dynamic output of a spin VCSEL from time-domain simulations, against supercritical bifurcation curves obtained by the standard continuation package AUTO. This helps us better understand the underlying dynamics of the spin VCSELs.
\end{abstract}

DOI: 10.1103/PhysRevA.96.013840

\section{INTRODUCTION}

Spintronics represents an exciting research field where the electron spin is used as an information carrier. Areas of interest include the development of novel devices as well as research concerning understanding and controlling the fundamental physical processes such as spin manipulation [1-3]. For a recent review of this field, we refer the reader to Ref. [4] and references therein.

As a specific example of spintronic devices, spin-polarized vertical-cavity surface-emitting lasers (VCSELs) have attracted considerable attention [5-9]. In such lasers, the output polarization is controlled by injecting spin-polarized electrons into the laser active region. Practical methods to do this involve the use of either magnetic contacts for electrical injection or carrier generation via optical pumping using circularly polarized light. Spin VCSELs are known to exhibit superior properties over conventional counterparts; examples include threshold reduction, spin control of the lasing output, and much faster dynamics [3]. The studies of such spin-controlled lasers may lead to a wide range of applications in areas such as optical communications, quantum computing, optical information processing and data storage, reconfigurable optical interconnects, and biomedical sensing [2,3,10-12].

Earlier experimental and theoretical studies on spin VCSELs have been focused on their steady-state operation [3,7,10,13-19]. For example, using optical pumping, switching in the output polarization between two opposite polarization states, i.e., left circularly polarized (LCP) and right circularly polarized (RCP), can be controlled via the pump polarization. In these studies, the output polarization ellipticity characterizing the degree of asymmetry of the emission is usually employed as an effective indicator. For example, by measuring the variation of output polarization ellipticity, the change in sign indicates the occurrence of a form of polarization switching [14,19]. To understand this kind of polarization switching, a combined numerical stability and asymptotic analysis of the rate equation model has been carried out by Susanto et al. [18]. This has demonstrated that the stability change of two possible sets of steady-steady (equilibria) solutions, termed in-phase and out-of-phase, may account for such switching behavior. Their asymptotic analysis, however, only provides qualitative agreement with numerical simulations for the case of optical pumping just above the threshold and very small optical pump ellipticity. Thus there remains the need for further work to provide clarity on the validity of the theoretical model for the full range of pump ellipticity and for higher values of optical pumping above threshold.

Very recently, Virte et al. have experimentally demonstrated, in a free-running, conventional VCSEL, the existence of polarization dynamics and chaos which originate from a nonlinear coupling between two elliptically polarized modes [20]. Bifurcation analysis based on the widely used spin-flip model (SFM) has also confirmed that such dynamical regimes could arise from the destabilization of the elliptically polarized states created by a pitchfork bifurcation on the lower-frequency linearly polarized state $[21,22]$. Likewise, various forms of instability could occur in free-running spin VCSELs, such as periodic oscillations and chaos. For VCSELs under continuous optical pump, Gahl et al. have predicted the possibility of self-sustained polarization oscillations with the mechanism being mainly associated with the cavity birefringence [23]. Gerhardt et al. have demonstrated experimentally in an 850-nm VCSEL that transient polarization oscillations can be generated using short pulsed optical spin injection [24]. They further reported experiments and SFM-based simulations on the frequency tuning of these polarization oscillations by applying mechanical strain to the VCSEL structure [25], where the frequencies are increased up to $44 \mathrm{GHz}$ and the tuning range is about $34 \mathrm{GHz}$. In our previous work, we have demonstrated self-sustained periodic oscillations that can be tuned from 8.6 to $11 \mathrm{GHz}$ with pump polarization in an optically pumped 1300-nm dilute nitride spin VCSEL [26]. Simulations using the SFM have achieved good agreement with the experimental 
results [27]. Additionally, rich instabilities in spin VCSELs have also been theoretically analyzed with the full SFM equations [28], where the largest Lyapunov exponent (LLE) is used to determine regions of stability and instability. The nonlinear polarization dynamics including chaos in spin VCSELs has been investigated experimentally and/or simulated theoretically, but here we examine and explain its bifurcations.

The objective of the present contribution is twofold. First, we conduct asymptotic analysis of the stability of the steadystate solutions of the spin VCSELs which extends the previous work of Susanto et al. [18]. Here we employ a different expansion using a scaling parameter different from those used before [18]. Using this, we find that our numerical and asymptotic results agree with each other both qualitatively and quantitatively for a pump of arbitrary ellipticity, even when the spin VCSEL is biased up to $50 \%$ above the lasing threshold. Second, we study bifurcations by using both direct numerical integration of the rate equations and the continuation technique. This allows tracking of the stable or unstable steady-state and periodic solutions and detection of various bifurcations. We show that the transition to optical chaos can occur following both a period-doubling route and a quasiperiodic route, depending on controllable parameters such as pump strength and polarization ellipticity. These also reveal insights into the range of different bifurcations in spin VCSELs, including Hopf bifurcations $(\mathrm{H})$, period-doubling bifurcations (PD), saddle-node of limit cycle bifurcations (LPC), and torus bifurcations (TR). A comparison between the web of these bifurcations and maps of dynamical regimes reveals the physical mechanism governing the underlying dynamics as well as the routes to chaos. In addition, the tunable hysteresis interval is identified with bifurcation analysis based on forward and backward sweep of the magnitude of the pump, indicating the coexistence of different dynamical states (e.g., bistability).

The paper is organized as follows. Section II presents the theoretical model for spin VCSELs. In Sec. III, we study the steady-state operation with the help of asymptotic analysis. Section IV is devoted to the bifurcation analysis, where different routes to chaos and various bifurcations are discussed. Finally, our conclusions are summarized in Sec. V.

\section{THE THEORETICAL MODEL}

Optically pumped spin VCSELs can be modeled by the following equations based on the extension of the well-known SFM [18,23,29-32]:

$$
\begin{gathered}
\frac{d \bar{E}_{+}}{d t}=\kappa(N+m-1)(1+i \alpha) \bar{E}_{+}-\left(\gamma_{a}+i \gamma_{p}\right) \bar{E}_{-}, \\
\frac{d \bar{E}_{-}}{d t}=\kappa(N-m-1)(1+i \alpha) \bar{E}_{-}-\left(\gamma_{a}+i \gamma_{p}\right) \bar{E}_{+}, \quad \text { (2) } \\
\frac{d N}{d t}=\gamma\left[\eta-\left(1+\left|\bar{E}_{+}\right|^{2}+\left|\bar{E}_{-}\right|^{2}\right) N-\left(\left|\bar{E}_{+}\right|^{2}-\left|\bar{E}_{-}\right|^{2}\right) m\right],
\end{gathered}
$$

$$
\begin{aligned}
\frac{d m}{d t}= & \gamma P \eta-\left[\gamma_{s}+\gamma\left(\left|\bar{E}_{+}\right|^{2}+\left|\bar{E}_{-}\right|^{2}\right)\right] m \\
& -\gamma\left(\left|\bar{E}_{+}\right|^{2}-\left|\bar{E}_{-}\right|^{2}\right) N
\end{aligned}
$$

where $\bar{E}_{+}$and $\bar{E}_{-}$represent the time-dependent variables of the RCP and LCP electric field components, respectively. The normalized carrier variables $N$ and $m$ are defined by $N=\left(n_{+}+n_{-}\right) / 2$ and $m=\left(n_{+}-n_{-}\right) / 2$, where $n_{+}$and $n_{-}$ are the corresponding normalized densities of electrons with spin-down and spin-up, respectively. Amplitude anisotropy is modeled through linear dichroism $\gamma_{a}$ and phase anisotropy is modeled by the linear birefringence $\gamma_{p}$. Other parameters are defined as follows: $\kappa$ is the optical field decay rate, $\gamma$ is the decay rate of $N, \gamma_{s}$ is the spin-flip relaxation rate, $\alpha$ is the linewidth enhancement factor, $\eta=\eta_{+}+\eta_{-}$is the total normalized pump power of the optical pumping $(\eta=1$ represents the pump threshold), and the pump polarization ellipticity $P$ is defined as

$$
P=\frac{\eta_{+}-\eta_{-}}{\eta_{+}+\eta_{-}},
$$

where $\left(\eta_{+}, \eta_{-}\right)$are dimensionless circularly polarized pump components that describe polarized optical pumping. Note that the equation is invariant under the transformation $P \rightarrow-P$, $m \rightarrow-m, E_{ \pm} \rightarrow E_{\mp}$. Therefore, without loss of generality, one may only consider the case of $P<0$.

\section{STEADY-STATE OPERATION}

In this section, we focus on the steady-state solutions and determine their stability through asymptotic analysis. These asymptotic results are compared to numerical results. We consider a particular set of parameter values that are comparable to those used in [18], and examine the continuous-wave (cw) characteristics of the spin VCSEL for wide regions of operational conditions. The following values of the parameters are chosen for the spin VCSEL: $\alpha=3, \gamma_{p}=150 \mathrm{~ns}^{-1}, \gamma_{a}=0$, $\gamma=1 \mathrm{~ns}^{-1}$, and $\kappa=250 \mathrm{~ns}^{-1}$. The pump strength $\eta$ is varied in the range of $\sim 1-2$, the pump ellipticity $P$ in the range of $\sim-1-0$, and the spin-flip relaxation rate $\gamma_{s}$ in the range of $\sim 10-150 \mathrm{~ns}^{-1}$.

Our analysis is particularly pertinent to time-independent solutions. In that case, we look for solutions in a rotating frame of the form

$$
\bar{E}_{+}=E_{+} e^{i \omega t}, \bar{E}_{-}=E_{-} e^{i \theta} e^{i \omega t}, \quad N=N_{s}, m=m_{s},
$$

with all the unknown variables, i.e., $E_{+}, E_{-}, \theta, \omega, N_{s}, m_{s}$, being time independent and real valued. When $\theta$ is the "continuation" of 0 or $\pi$, we refer to the solution as in-phase or out-of-phase, respectively.

The linear stability of the time-independent solution is obtained by substituting $\bar{E}_{+}=\left(E_{+}+\epsilon \bar{E}_{+} e^{\lambda t}\right) e^{i \omega t}, \bar{E}_{-}=$ $\left(E_{-} e^{i \theta}+\epsilon \widehat{E}_{-} e^{\lambda t}\right) e^{i \omega t}, N=N_{s}+\epsilon \widehat{N} e^{\lambda t}, m=m_{s}+\epsilon \widehat{m} e^{\lambda t}$ into the governing equations and linearizing for small $\epsilon$ to obtain the eigenvalue problem

$$
\mathcal{M} \underline{v}=\lambda \underline{v},
$$

where $\underline{v}=\left(\widehat{E}_{+}, \widehat{E}_{-}, \widehat{E}_{+}^{*}, \widehat{E}_{-}^{*}, \widehat{N}, \widehat{m}\right)^{T}, \mathbf{a}^{T}$ denotes the transpose of the matrix a, and $*$ represents complex conjugation. For the coefficient matrix $\mathcal{M}$, we refer to Eq. (9) in [18] (note that this matrix can also be found in Appendix A for convenience).

The governing equations (1)-(4) and (6) are solved using a Newton-Raphson method. The stability of the in-phase 
and out-of-phase solutions are then determined by solving the eigenvalue problem (7). We obtain six eigenvalues $\left(\lambda_{1}, \lambda_{2}, \ldots, \lambda_{6}\right)$ in total, and one $\left(\lambda_{6}\right)$ of them is always zero, which is associated with the arbitrariness of the phase of the electric field. These eigenvalues can be ordered as

$$
\operatorname{Re}\left[\lambda_{5}\right] \leqslant \operatorname{Re}\left[\lambda_{4}\right] \leqslant \operatorname{Re}\left[\lambda_{3}\right] \leqslant \operatorname{Re}\left[\lambda_{2}\right] \leqslant \operatorname{Re}\left[\lambda_{1}\right],
$$

where there are two complex conjugate pairs $\left(\lambda_{1}, \lambda_{2}\right)$ and $\left(\lambda_{3}\right.$, $\left.\lambda_{4}\right)$. Laser stability is determined by the least damped solution under small perturbations. In other words, eigenvalue(s) $\lambda_{n}$ with the lowest $n$ or the largest $\operatorname{Re}\left[\lambda_{n}\right]$ is (are) the so-called critical one(s) according to the assignment in Eq. (8).

In the following, we derive asymptotic expressions of the steady-state solutions using an approach similar to that described in [18]. However, the small parameter for expansion chosen here is completely different, leading to much better approximate solutions, as we will show below. For the particular set of parameter values used in the numerical computations, we scale the parameters as

$$
\gamma_{p}=\tilde{\gamma}_{p} / \varepsilon^{2}, \kappa=\tilde{\kappa} / \varepsilon^{2}, \gamma_{s}=\tilde{\gamma}_{s} / \varepsilon^{2},
$$

where $|\varepsilon| \ll 1$ is a scaling parameter. For simplicity of our analysis, we normalize the key laser parameters in units of the electron density decay rate $\gamma$ and assume the other parameters as $O(1)$. Note that the reason why we only deal with the three parameters in Eq. (9) is because they are usually much larger than other parameters of the SFM [17,18,21,23,28].

We then write the dependent variables in asymptotic series. Performing the asymptotic expansions and computing the governing equation at every order of $\varepsilon$, we obtain that the in-phase solution is given asymptotically by

$$
\begin{gathered}
E_{+}=\frac{\sqrt{2}}{2} \sqrt{\eta-1}-\sqrt{2} \varepsilon^{2} \frac{\sqrt{\eta-1} \tilde{\kappa} \alpha \gamma \eta P}{4 \tilde{\gamma}_{p} \tilde{\gamma}_{s}}+O\left(\varepsilon^{4}\right) \\
E_{-}=\frac{\sqrt{2}}{2} \sqrt{\eta-1}+\sqrt{2} \varepsilon^{2} \frac{\sqrt{\eta-1} \tilde{\kappa} \alpha \gamma \eta P}{4 \tilde{\gamma}_{p} \tilde{\gamma}_{s}}+O\left(\varepsilon^{4}\right) \\
\theta=-\varepsilon^{2} \frac{\tilde{\kappa} \gamma \eta P}{\tilde{\gamma}_{p} \tilde{\gamma}_{s}}+\varepsilon^{4} \frac{\tilde{\kappa} \gamma^{2} \eta P\left(\tilde{\kappa} \alpha-\tilde{\gamma}_{p}\right)(1-\eta)}{\tilde{\gamma}_{p}^{2} \tilde{\gamma}_{s}^{2}}+O\left(\varepsilon^{6}\right) \\
\omega=-\tilde{\gamma}_{p} / \varepsilon^{2}+\frac{\varepsilon^{2}}{2} \frac{(\tilde{\kappa} \gamma \eta P)^{2}\left(\alpha^{2}+1\right)}{\tilde{\gamma}_{s}^{2} \tilde{\gamma}_{p}}+O\left(\varepsilon^{4}\right) \\
N_{s}=1+\varepsilon^{4} \frac{\tilde{\kappa} \alpha P^{2} \eta^{2} \gamma^{2}}{\tilde{\gamma}_{s}^{2} \tilde{\gamma}_{p}}+O\left(\varepsilon^{6}\right) \\
m_{s}=\varepsilon^{2} \frac{\gamma_{\eta} P}{\tilde{\gamma}_{s}}-\varepsilon^{4} \frac{(\eta-1) \gamma^{2} \eta P\left(\tilde{\gamma}_{p}-\tilde{\kappa}^{2} \alpha\right)}{\tilde{\gamma}_{p} \tilde{\gamma}_{s}^{2}}+O\left(\varepsilon^{6}\right)
\end{gathered}
$$

Next, to solve the eigenvalue problem (7), it is then natural to also expand the stability matrix, the eigenvalues, and the corresponding eigenvectors in $\varepsilon$ as

$$
\begin{aligned}
\mathcal{M} & =\mathcal{M}_{-2} / \varepsilon^{2}+\mathcal{M}_{0}+\mathcal{M}_{2} \varepsilon+\cdots, \\
\underline{v} & =\underline{v}_{0}+\underline{v}_{2} \varepsilon+\cdots, \\
\lambda & =\lambda_{-2} / \varepsilon^{2}+\lambda_{0}+\lambda_{2} \varepsilon+\cdots .
\end{aligned}
$$

Performing the same calculations as in [18], we obtain that the critical eigenvalue responsible for the instability is given asymptotically up to $O\left(\varepsilon^{4}\right)$ by

$$
\begin{aligned}
\lambda= & \frac{2 i \tilde{\gamma}_{p}}{\varepsilon^{2}}+\frac{\tilde{\kappa} \gamma(\alpha-i)(1-\eta)}{2 \tilde{\gamma}_{p}-i \tilde{\gamma}_{s}} \\
& +\frac{\varepsilon^{2} \gamma^{2} \tilde{\kappa}(i-\alpha) \sum_{j=0}^{3} K_{j} \tilde{\gamma}_{s}^{j}}{4 i \tilde{\gamma}_{s}^{2} \tilde{\gamma}_{p}\left(2 \tilde{\gamma}_{p}-i \tilde{\gamma}_{s}\right)^{5}}
\end{aligned}
$$

with

$$
\begin{aligned}
K_{3}= & \left(\tilde{\kappa}+4 i \tilde{\gamma}_{p}-i \tilde{\kappa} \alpha\right)\left(1-2 \eta+\eta^{2}\right)+4 \tilde{\kappa} P^{2}(1+i \alpha) \eta^{2}, \\
K_{2}= & \left(6 \tilde{\gamma}_{p} \tilde{\kappa} \alpha-2 i \tilde{\gamma}_{p} \tilde{\kappa}-8 \tilde{\gamma}_{p}^{2}\right)\left(1-2 \eta+\eta^{2}\right) \\
& +24 \tilde{\gamma}_{p} \tilde{\kappa} P^{2}(i-\alpha) \eta^{2}, \\
K_{1}= & -48 \tilde{\gamma}_{p}^{2} \tilde{\kappa} P^{2}(1+i \alpha) \eta^{2}, \\
K_{0}= & -32 \tilde{\gamma}_{p}^{3} \tilde{\kappa} P^{2}(i-\alpha) \eta^{2} .
\end{aligned}
$$

Performing the same calculations for the out-of-phase equilibrium, we obtain that the solution is asymptotically given by

$$
\begin{aligned}
& E_{+}=\frac{\sqrt{2}}{2} \sqrt{\eta-1}+\sqrt{2} \varepsilon^{2} \frac{\sqrt{\eta-1} \tilde{\kappa} \alpha \gamma \eta P}{4 \tilde{\gamma}_{p} \tilde{\gamma}_{s}}+O\left(\varepsilon^{4}\right), \\
& E_{-}=\frac{\sqrt{2}}{2} \sqrt{\eta-1}-\sqrt{2} \varepsilon^{2} \frac{\sqrt{\eta-1} \tilde{\kappa} \alpha \gamma \eta P}{4 \tilde{\gamma}_{p} \tilde{\gamma}_{s}}+O\left(\varepsilon^{4}\right), \\
& \theta=\pi+\varepsilon^{2} \frac{\tilde{\kappa} \gamma \eta P}{\tilde{\gamma}_{p} \tilde{\gamma}_{s}}+\varepsilon^{4} \frac{\tilde{\kappa} \gamma^{2} \eta P\left(\tilde{\kappa} \alpha+\tilde{\gamma}_{p}\right)(1-\eta)}{\tilde{\gamma}_{p}^{2} \tilde{\gamma}_{s}^{2}}+O\left(\varepsilon^{6}\right), \\
& \omega=\tilde{\gamma}_{p} / \varepsilon^{2}-\frac{\varepsilon^{2}}{2} \frac{(\tilde{\kappa} \gamma \eta P)^{2}\left(\alpha^{2}+1\right)}{\tilde{\gamma}_{s}^{2} \tilde{\gamma}_{p}}+O\left(\varepsilon^{4}\right), \\
& N_{s}=1-\varepsilon^{4} \frac{\tilde{\kappa} \alpha P^{2} \eta^{2} \gamma^{2}}{\tilde{\gamma}_{s}^{2} \tilde{\gamma}_{p}}+O\left(\varepsilon^{6}\right), \\
& m_{s}=\varepsilon^{2} \frac{\gamma \eta P}{\tilde{\gamma}_{s}}-\varepsilon^{4} \frac{(\eta-1) \gamma^{2} \eta P\left(\tilde{\gamma}_{p}+\tilde{\kappa} \alpha\right)}{\tilde{\gamma}_{p} \tilde{\gamma}_{s}^{2}}+O\left(\varepsilon^{6}\right),
\end{aligned}
$$

from which we obtain that up to $O\left(\varepsilon^{4}\right)$, the critical eigenvalue is given by

$$
\begin{aligned}
\lambda= & \frac{2 i \tilde{\gamma}_{p}}{\varepsilon^{2}}+\frac{\tilde{\kappa} \gamma(\alpha+i)(\eta-1)}{2 \tilde{\gamma}_{p}-i \tilde{\gamma}_{s}} \\
& +\frac{\varepsilon^{2} \gamma^{2} \tilde{\kappa}(i+\alpha) \sum_{j=0}^{3} K_{j} \tilde{\gamma}_{s}^{j}}{4 i \tilde{\gamma}_{s}^{2} \tilde{\gamma}_{p}\left(2 \tilde{\gamma}_{p}-i \tilde{\gamma}_{s}\right)^{5}}
\end{aligned}
$$

with

$$
\begin{aligned}
K_{3}= & \left(\tilde{\kappa}+4 i \tilde{\gamma}_{p}+i \tilde{\kappa} \alpha\right)\left(1-2 \eta+\eta^{2}\right)+4 \tilde{\kappa} P^{2}(1-i \alpha) \eta^{2}, \\
K_{2}= & -\left(6 \tilde{\gamma}_{p} \tilde{\kappa} \alpha+2 i \tilde{\gamma}_{p} \tilde{\kappa}+8 \tilde{\gamma}_{p}^{2}\right)\left(1-2 \eta+\eta^{2}\right) \\
& +24 \tilde{\gamma}_{p} \tilde{\kappa} P^{2}(i+\alpha) \eta^{2}, \\
K_{1}= & -48 \tilde{\gamma}_{p}^{2} \tilde{\kappa} P^{2}(1-i \alpha) \eta^{2}, \\
K_{0}= & 32 \tilde{\gamma}_{p}^{3} \tilde{\kappa} P^{2}(i+\alpha) \eta^{2} .
\end{aligned}
$$

The above analysis is carried out at $\gamma_{a}=0$, which is justified because, in most cases in the VCSEL system, the amplitude anisotropy is much smaller compared to the phase 


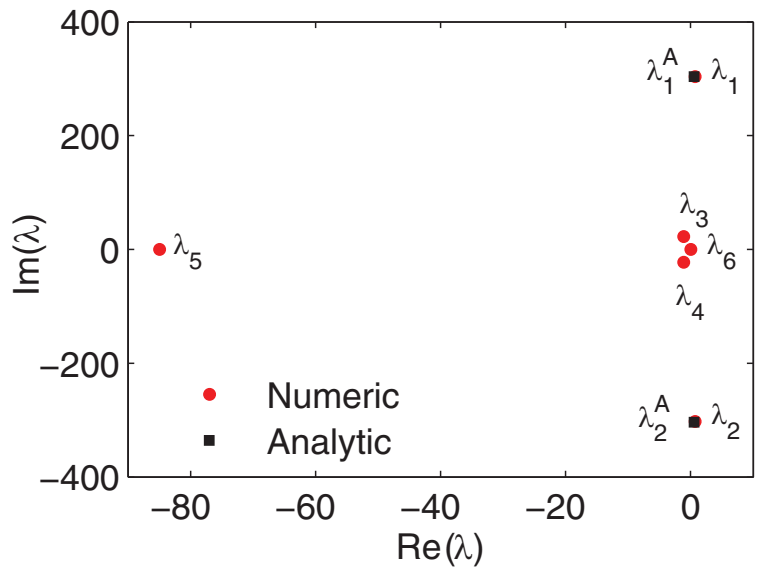

FIG. 1. Eigenvalues of the spin-polarized VCSEL equilibrium in the complex plane for $\gamma_{s}=80 \mathrm{~ns}^{-1}, \eta=2$, and $P=-1$. Red dots stand for numerical results, while black squares represent approximate results of the critical eigenvalues.

anisotropy, i.e., $\gamma_{a} \ll \gamma_{p}$. It should be noted that the present method also works even though a nonzero value of $\gamma_{a}$ is considered. Besides, at order $\varepsilon^{0}$, Eqs. (16) and (23) accounting for the critical eigenvalues can be reduced to an earlier approximation by Mulet $e t a l$. in the limit of $P=0$ and large $\gamma_{s}$ (see Eq. (5) in [33] and the paragraph of text following the equation).

As an example, in Fig. 1 numerical results of the six eigenvalues for the spin-VCSEL model, denoted with $\lambda_{1}, \lambda_{2}, \ldots$, and $\lambda_{6}$, are plotted with circles and the approximate results of the critical eigenvalues, denoted with $\lambda_{1}^{A}$ and $\lambda_{2}^{A}$, are plotted with squares. One can see that the asymptotic results give a good approximation of the accurate numerical results, giving us confidence for further comparison.

A question one may ask is how approximate solutions behave when a certain device parameter is varied. Specifically, we consider one key parameter of the spin VCSELs, i.e., the spin-flip relaxation rate $\gamma_{s}$. To answer this question, we present the variations of real and imaginary parts of the critical eigenvalue as a function of $\gamma_{s}$ for $\eta=2$ and $P=-1$ in Fig. 2, where the numerics and the asymptotic results in both in-phase and out-of-phase cases are included. It is observed that our asymptotic curves always follow those of numerical results; however, the quantitative differences between them are quite evident. One can also see that the approximation is getting better when $\gamma_{s} \sim O\left(1 / \varepsilon^{2}\right)$, which is expected.

An interesting question is whether the approximate results can be improved by changing controllable parameters such as pump strength $\eta$ and pump ellipticity $P$. This question can be answered by comparing the results shown in Fig. 3 for different sets of $\eta$ and $P$. Here only the real parts of the critical eigenvalues are shown, since their sign determines the stability of the corresponding equilibrium instead of the imaginary parts. First, we decrease $\eta$ from 2 to 1.5 and show in Figs. 3(a1)-3(d1) real parts of the critical eigenvalues of in-phase and out-of-phase solutions as a function of $\gamma_{s}$, when $P$ is fixed at $P=-1, P=-0.8, P=-0.6$, and $P=-0.4$. The asymptotic lines resulting from (16) and (23) (shown as dashed lines) are approximations to the real part curves of the

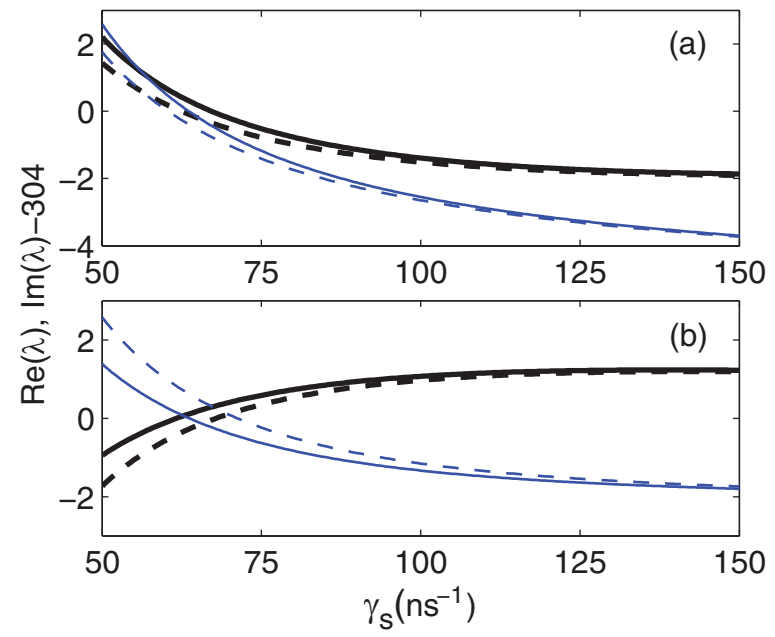

FIG. 2. The real part (thick) and the imaginary part (thin) of the critical eigenvalues as a function of $\gamma_{s}$ for the (a) in-phase and (b) out-of-phase equilibrium. The dashed lines are the asymptotic approximations (16) and (23). Parameters are the same as those in Fig. 1.

critical eigenvalues. The exact numerical curves are shown as solid lines in these figures. A comparison of Figs. 2 and 3(a1) reveals that the accuracy of the approximate results is improved at the lower pump level $(\eta=1.5)$. However, a distinction can still be made between approximate and numerical results. The comparison among Figs. 3(a1)-3(d1) reveals that decreasing $|P|$ greatly improves the approximate results, particularly when $|P| \leqslant 0.6$, where the curves for analytical and numerical results become almost superimposed both for in-phase and out-of-phase cases. Second, a further decrease in $\eta$ leads to more accurate approximate results which are in surprisingly good agreement with numerical solutions for the full range of $P$; for example, see Figs. 3(a2)-3(d2) and Figs. 3(a3)-3(d3) for $\eta=1.2$ and $\eta=1.1$, respectively.

In addition to those comparisons mentioned above, other interesting features revealed in Fig. 3 can be summarized as follows. First, decreasing pump polarization $|P|$ or increasing spin-flip relaxation rate $\gamma_{s}$ tends to stabilize the in-phase solution. Second, increasing $\gamma_{s}$ induces a switching behavior between the onset of stable in-phase and out-of-phase solutions for large enough $|P|$; the critical value of $\gamma_{s}$ at this polarization switching point can be tuned via either the pump polarization ellipticity or magnitude, as found in preliminary results reported in $[14,19,26]$. Third, for all values of $\eta$ in Fig. 3, the system always admits the in-phase solution and thus no switching is observed over the interval of $\gamma_{s}$ considered, when $|P|$ is small enough, roughly $|P| \leqslant 0.4$. We note that no instability is found for these pump levels, indicating such parameter sets considered are appropriate for stability analysis as well as comparing asymptotic and numerical results.

We would like to stress that the spin-VCSEL system may lose stability at an interval of smaller values of $\gamma_{s}$ for higher pump levels and the present approximation is not good enough for predicting instabilities via the Hopf bifurcation in this situation. As indicated in Fig. 2, the approximation underestimates the real parts of the critical eigenvalue both for in-phase and out-of-phase solutions. However, the approximation of the 


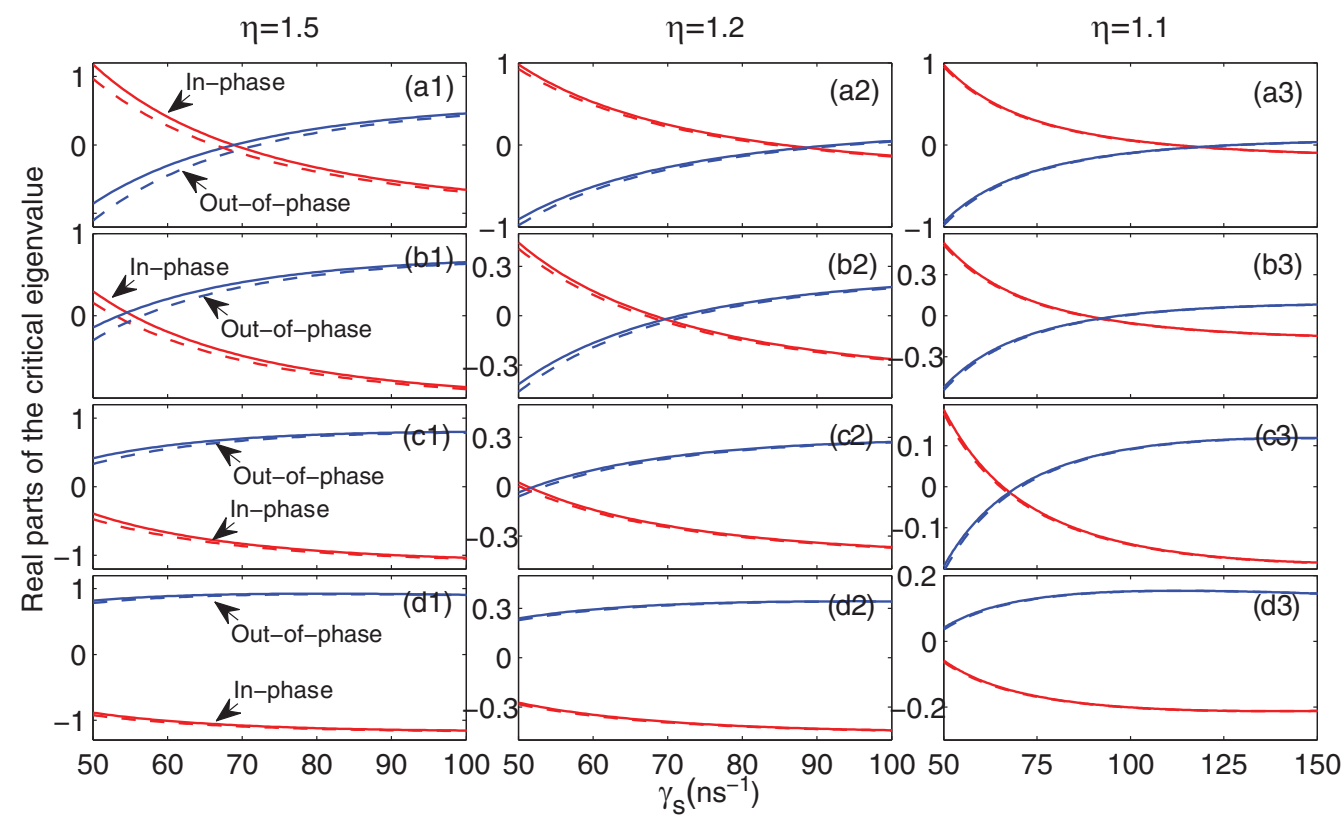

FIG. 3. The real part of the critical eigenvalues of the in-phase (red) and out-of-phase (blue) equilibrium as a function of $\gamma_{s}$, for different values of $\eta$ and $P$. (a1)-(a3) $P=-1$, (b1)-(b3) $P=-0.8$, (c1)-(c3) $P=-0.6$, and (d1)-(d3) $P=-0.4$. (a1)-(d1) $\eta=1.5$, (a2)-(d2) $\eta=1.2$, and (a3) $-(\mathrm{d} 3) \eta=1.1$. The dashed lines are the asymptotic approximations (16) and (23).

oscillation frequency is very close to the accurate estimation from simulations (determined by the imaginary part of the critical eigenvalue), as shown in Fig. 4. It is worth noting that the frequency of such continuous birefringence-induced oscillation is mainly determined by the birefringence rate $\gamma_{p}$ (called birefringence frequency $\gamma_{p} / \pi=47.75 \mathrm{GHz}$ ), which is very different from the situation for the conventional laser diodes. For example, in a conventional semiconductor laser with external perturbation, relaxation oscillations may become undamped through a Hopf bifurcation and give rise to a selfsustained limit-cycle solution at the frequency of the relaxation oscillation $(\sim 3.6 \mathrm{GHz}$ for the set of parameter values used in Fig. 2). As we stated in Sec. I, we are dealing with a very special VCSEL which may allow for faster dynamics; see our earlier

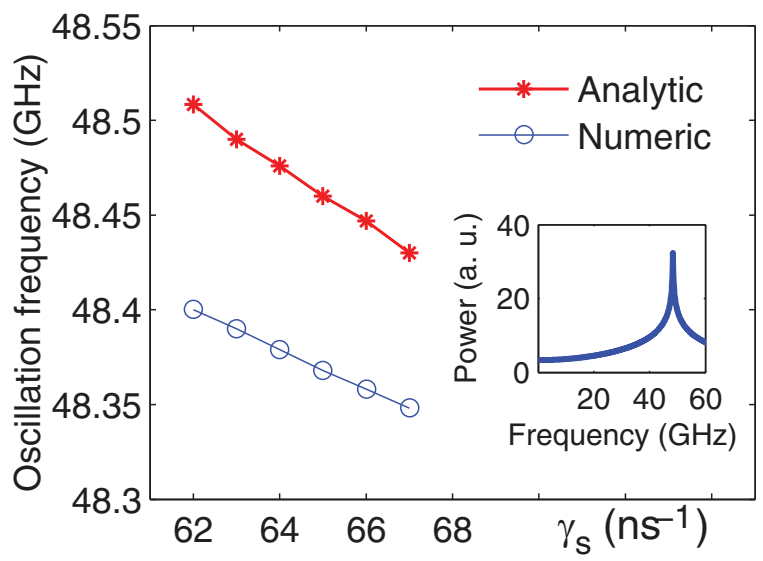

FIG. 4. An example of comparison between the approximated and simulated oscillation frequency as a function of $\gamma_{s}$, with (inset) the rf spectrum giving the evidence of period one oscillation. Parameters are the same as those in Fig. 2. work [34] for further details. We argue that it is practically impossible to reproduce all features of the numerical findings using the current approximation method, so in the following section we will consider higher pump levels and reveal the nature of the rich dynamics by means of simulation. To this end, we vary some of the key parameters and explore various bifurcations.

\section{BIFURCATION SCENARIOS}

We report here the use of bifurcation analysis to identify the various forms of instability predicted by the SFM. Oneparameter bifurcations are calculated by direct numerical integration of Eqs. (1)-(4), while for the two-parameter bifurcations obtained by the standard continuation package AUTO [35], these original equations cannot be directly used because any solution of Eqs. (1)-(4) can be multiplied by a common phase factor in both RCP and LCP electric field components, leading to an $S^{1}$ symmetry [36]. That is,

$$
\left(\bar{E}_{+}, \bar{E}_{-}, N, m\right) \rightarrow\left(e^{i \phi} \bar{E}_{+}, e^{i \phi} \bar{E}_{-}, N, m\right), \phi \in[0,2 \pi) .
$$

To remove the $S^{1}$ symmetry, one usually rewrites Eqs. (1)-(4) in terms of five equations, including two for the RCP and LCP electric field amplitudes, one for the phase difference, and two for the two carrier variables [21]. However, the phase difference equation contains terms consisting of the ratio between the RCP and LCP electric field amplitudes, and this may induce a singularity when one of them becomes very close to zero during the oscillation operation (as will be shown, for example, in Fig. 5), which makes numeric continuation software problematic.

In order to use the continuation package AUTO, we perform a variable transformation and obtain reduced coordinate equations, which we believe can solve the two problems 


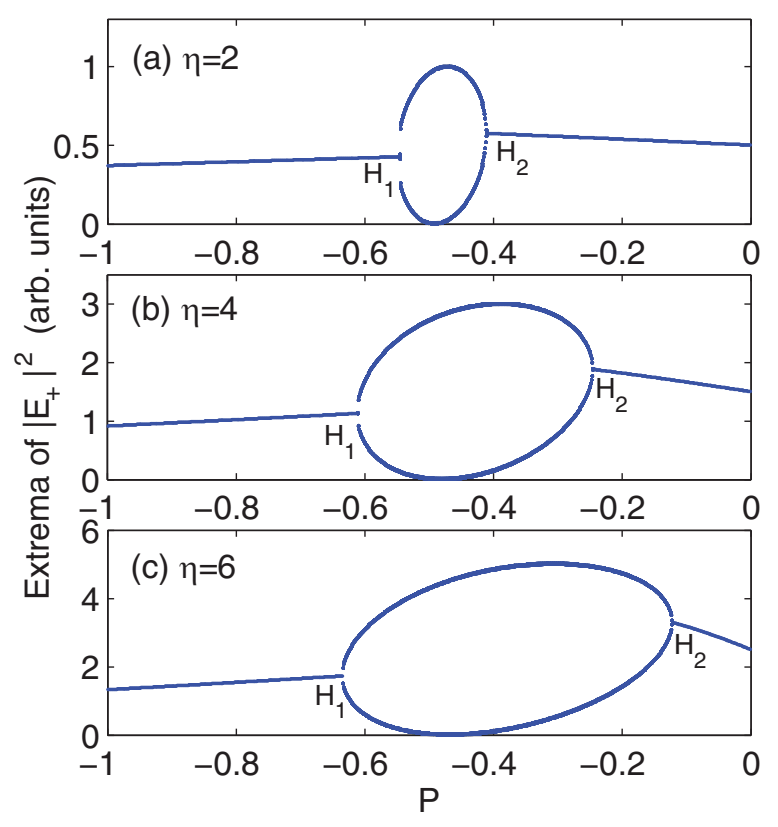

FIG. 5. Bifurcation diagrams of the RCP intensity as a function of $P$ for (a) $\eta=2$, (b) 4 , and (c) $6 . \mathrm{H}_{1}$ and $\mathrm{H}_{2}$ denote two different Hopf bifurcations. The spin-flip relaxation rate $\gamma_{s}=30 \mathrm{~ns}^{-1}$.

mentioned above. Specifically, two variables are defined as (see Appendix B for more details)

$$
\begin{gathered}
\Omega=\left|\bar{E}_{+}\right|^{2}-\left|\bar{E}_{-}\right|^{2}, \\
Q=\bar{E}_{-} \bar{E}_{+}^{*} .
\end{gathered}
$$

Rewriting the complex variable $Q$ in terms of real and imaginary parts as $Q=Q_{R}+i Q_{I}$, the rate equations become

$$
\begin{gathered}
\frac{d \Omega}{d t}=2 \kappa(N-1) \Omega+2 \kappa m \sqrt{\Omega^{2}+4|Q|^{2}}+4 \gamma_{p} Q_{I}, \\
\frac{d Q_{R}}{d t}=2 \kappa\left[Q_{R}(N-1)+\alpha m Q_{I}\right]-\gamma_{a} \sqrt{\Omega^{2}+4|Q|^{2}}, \\
\frac{d Q_{I}}{d t}=2 \kappa\left[Q_{I}(N-1)-\alpha m Q_{R}\right]-\gamma_{p} \Omega, \\
\frac{d N}{d t}=\gamma\left[\eta-\left(1+\sqrt{\Omega^{2}+4|Q|^{2}}\right) N-\Omega m\right], \\
\frac{d m}{d t}=\gamma P \eta-\left(\gamma_{s}+\gamma \sqrt{\Omega^{2}+4|Q|^{2}}\right) m-\gamma \Omega N,
\end{gathered}
$$

where $|Q|^{2}=\left|Q_{R}\right|^{2}+\left|Q_{I}\right|^{2}$. In the following, this reduced system will be used in AUTO to obtain a comprehensive overview of the bifurcations involved in the spin VCSELs.

First we set the spin-flip relaxation rate $\gamma_{s}$ to $30 \mathrm{~ns}^{-1}$ and show one-parameter bifurcations by scanning the pump polarization ellipticity $P$. All other parameters are kept at the values used in Fig. 1. Figure 5 illustrates bifurcation diagrams, representing the minima and maxima (extrema) of RCP intensity $\left|\bar{E}_{+}\right|^{2}$, for three different values of the magnitude of the optical pump $\eta$. In all cases, the out-of-phase stationary state is always stable for large $|P|$ but becomes unstable via a Hopf bifurcation $\mathrm{H}_{1}$. It corresponds to the excitation of self-sustained polarization oscillations [period-

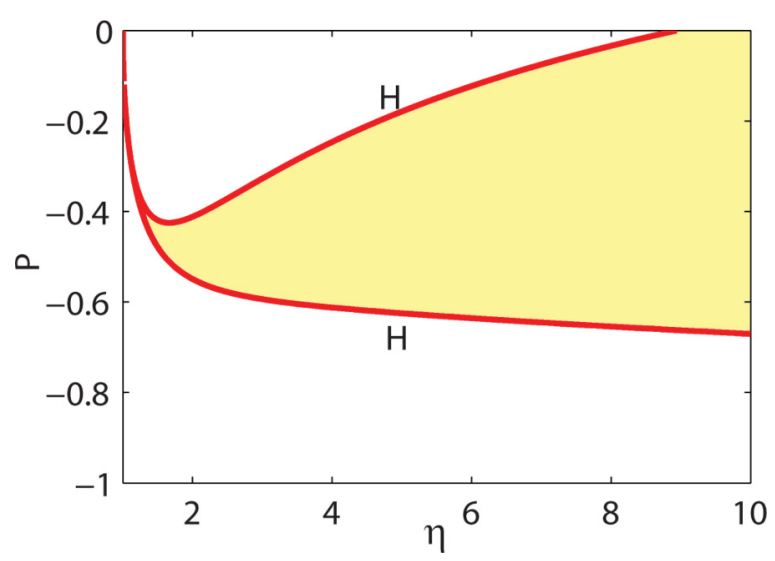

FIG. 6. The bifurcation diagram in the $(\eta, P)$ plane. The periodone oscillation region is highlighted in yellow. The spin-flip relaxation rate $\gamma_{s}=30 \mathrm{~ns}^{-1}$ and all other parameters are the same as those in the preceding section.

one (P1) dynamics] with a frequency mainly governed by the cavity birefringence (a detailed discussion related to this P1 oscillation can be found in [34]). A further decrease in $|P|$ from the instability range leads to a transition so that the in-phase stationary state takes over the stability from the oscillatory behavior. This corresponds to an inverse Hopf bifurcation $\mathrm{H}_{2}$. It is worth noting that increasing $\eta$ causes no additional bifurcations, but the finite interval of $P$ within which the P1 oscillation is possible is broadened.

Figure 6 shows the bifurcation diagram of Eqs. (27)-(31) in the plane of $\eta$ and $P$, consisting of only two Hopf bifurcations $\left(\mathrm{H}_{1}\right.$ and $\left.\mathrm{H}_{2}\right)$. The two Hopf curves are nearly superimposed in the low pump region and become separated with increasing $\eta$, leading to a broadening of the instability region. It is worth noting here that the overall shape of the P1 region in Fig. 6 is similar to some of those presented in [28], especially Fig. 5(b) of [28], although somewhat different parameter values were used. These results use the continuation technique for acquiring this stability map, whereas the LLE was used in [28]. Furthermore, we see no additional bifurcation curves here, in good agreement with one-parameter bifurcations obtained by direct numerical integration in Fig. 5. It should be noted that there exists a generalized (Bautin) Hopf bifurcation (not shown) on each Hopf curve when they start to approach each other in the low pump region. At this codimension-2 bifurcation point, the supercritical Hopf bifurcation becomes subcritical [37], thus no stable period orbit can be found when crossing the Hopf curve.

The reduced set of equations used here allows a systematic bifurcation study of the spin VCSELs, which greatly facilitates our understanding of possible switching transitions between various dynamical regimes. Of particular interest are further, complex bifurcation scenarios from Hopf bifurcation points. However, the present parameter values only allow for the steady and P1 states. For this reason, we now consider the following values of the parameters: $\kappa=230 \mathrm{~ns}^{-1}, \gamma_{s}=30$ or $60 \mathrm{~ns}^{-1}, \gamma_{p}=8.8 \pi \mathrm{ns}^{-1}, \gamma=0.68 \mathrm{~ns}^{-1}, \gamma_{a}=0$, and $\alpha=4$, and choose $\eta$ and $P$ as bifurcation parameters.

Consider $\gamma_{s}=30 \mathrm{~ns}^{-1}$. The resulting one-parameter bifurcation diagrams for increasing pump strength $\eta$ for this value of 

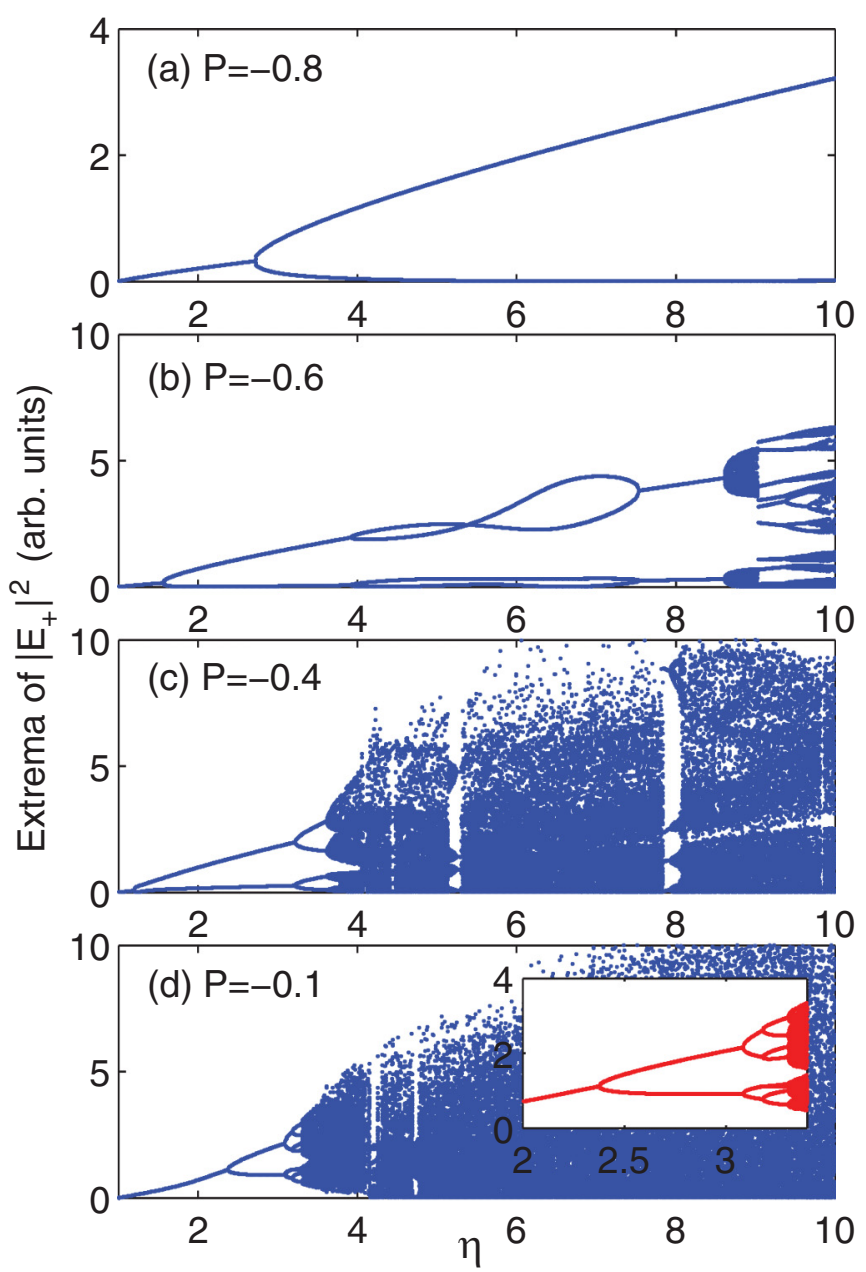

FIG. 7. Bifurcation diagram of the RCP intensity as a function of $\eta$ for (a) $P=-0.8$, (b) -0.6 , (c) -0.4 , and (d) -0.1 . The spinflip relaxation rate $\gamma_{s}=30 \mathrm{~ns}^{-1}$. Other parameters are specified in the text. The inset in (d) is a magnification of the period-doubling bifurcation region.

$\gamma_{s}$ are depicted in Fig. 7, where four values of pump ellipticity $P$ are considered. For $P=-0.8$ shown in Fig. 7(a), the system only goes through a stable steady state and P1 oscillation, similar to the case shown in Fig. 6. A decrease of $|P|$ leads to the appearance of more interesting bifurcations and thus unstable dynamical regimes. For example, when $P=-0.6$, the bifurcation diagram in Fig. 7(b) displays a very rich dynamics: P1, period two (P2), quasiperiodicity, and higher periodic oscillations. In this case, Hopf bifurcation, forward period-doubling bifurcation, backward period-doubling bifurcation, and torus bifurcation (not depicted in the figure) can be identified as $\eta$ is increased from the laser threshold. However, no transition to chaos is found. For a further decrease in $|P|$, varying $\eta$ makes the system undergo Hopf bifurcation, perioddoubling bifurcation, torus bifurcation, and further intricate bifurcations to chaos. Note that even after the system evolves to chaotic dynamics, windows of periodicity and quasiperiodicity are observed for different values of $\eta$. For very small values of $|P|$, the spin VCSEL becomes unstable and chaotic solely via a period-doubling route bifurcation. An example of such a bifurcation diagram is shown in Fig. 7(d) for $P=-0.1$.

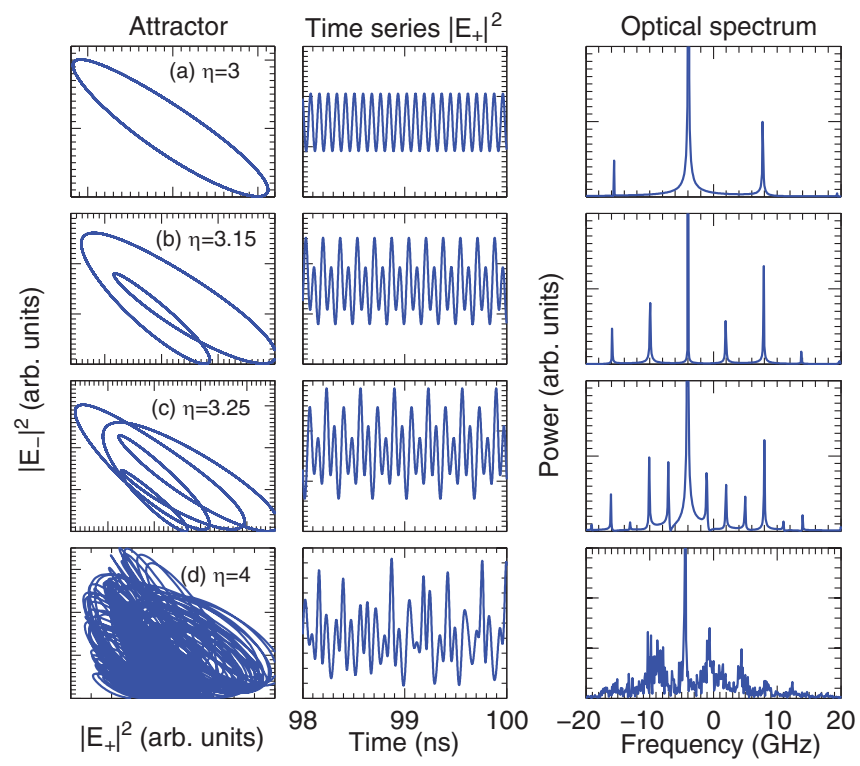

FIG. 8. Period-doubling route to chaos; $\gamma_{s}=30 \mathrm{~ns}^{-1}, P=-0.1$ and, from (a) to (d), $\eta=3,3.15,3.25$, and 4 , respectively.

As we stretch the horizontal axis in the inset, we clearly observe a successive period-doubling route to chaos. Some insight into the well-known period-doubling route to chaos is illustrated in Fig. 8, consisting of the attractor projected onto the plane of RCP and LCP intensities, the time series of RCP intensity, and the corresponding optical spectrum. As can be seen, an attracting periodic orbit Fig. 8(a) undergoes successive period-doublings [Figs. 8(b) and 8(c)], giving rise to chaotic dynamics [Fig. 8(d)]. The attractor and spectrum in Fig. 8(d) already indicate the appearance of typical features of chaos, which can be further confirmed by calculating the LLE [28,38-40], i.e., a positive LLE indicates the presence of chaos.

An entirely different route to chaos (i.e., the so-called quasiperiodic or breakup of a torus route to chaos [41,42]) can be found in the spin VCSEL with increasing $P$ at large values of $\eta$ for the particular set of parameter values used. An example is shown in Figs. 9 and 10, where $\eta=8.5$. We see

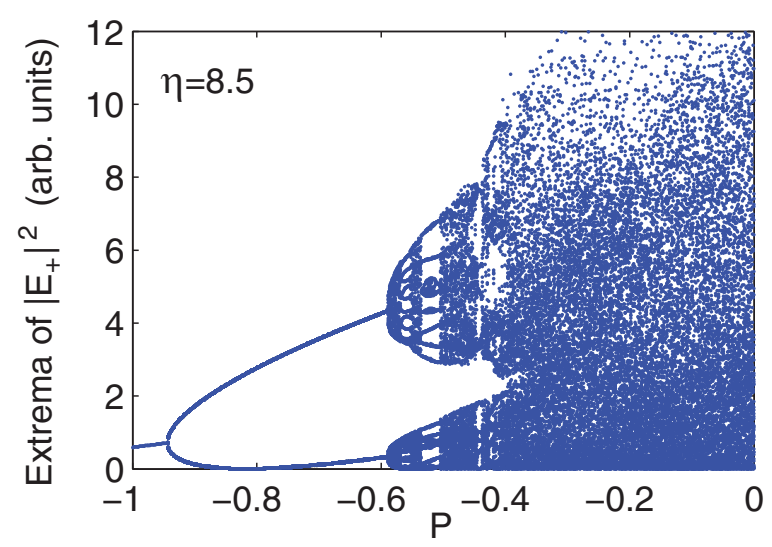

FIG. 9. Bifurcation diagram of the RCP intensity as a function of $P$ for $\eta=8.5$. Other parameters are the same as those in Fig. 6 . 

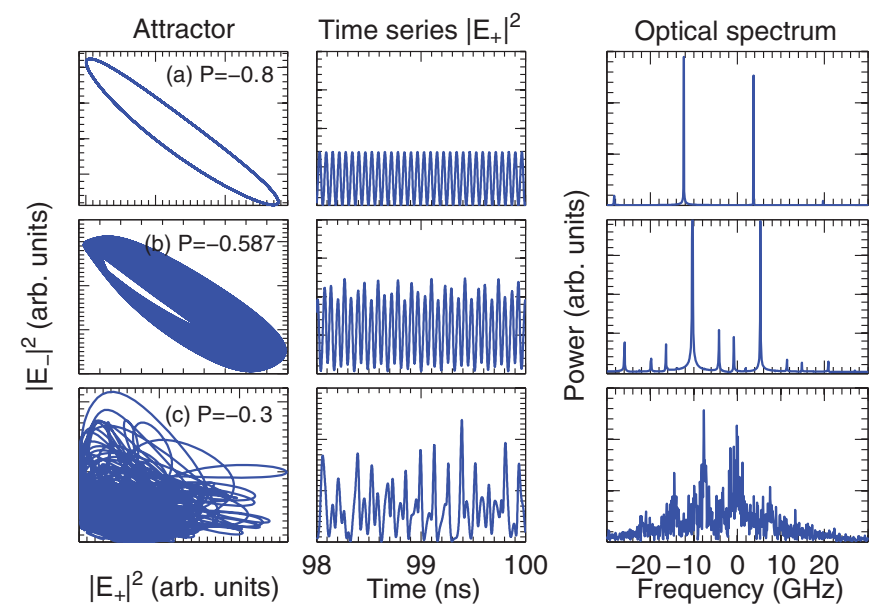

FIG. 10. Quasiperiodic route to chaos; $\eta=8.5$ and, from (a) to (c), $P=-0.8,-0.587$, and -0.3 , respectively.

that an attracting periodic orbit [Fig. 10(a)] loses its stability in a torus and quasiperiodic oscillations appear [Fig. 10(b)] (sometimes the bifurcation from a limit-cycle solution to a quasiperiodic solution is denoted as a secondary Hopf solution since it corresponds to a closed curve in the Poincaré section [42]). The breakup of the torus leads to chaotic motion [Fig. 10(c)].

Figure 11(a) illustrates a two-parameter bifurcation in the plane of $\eta$ and $P$ for $\gamma_{s}=30 \mathrm{~ns}^{-1}$, giving a comprehensive overview of the bifurcations. In particular, the map contains a web of curves for Hopf $(\mathrm{H})$, period-doubling $\left(\mathrm{PD}^{1}, \mathrm{PD}^{2}\right)$, torus (TR), and saddle-node of limit cycles (LPC). Here, $\mathrm{PD}^{1}$ and $\mathrm{PD}^{2}$ denote two successive period doublings of a
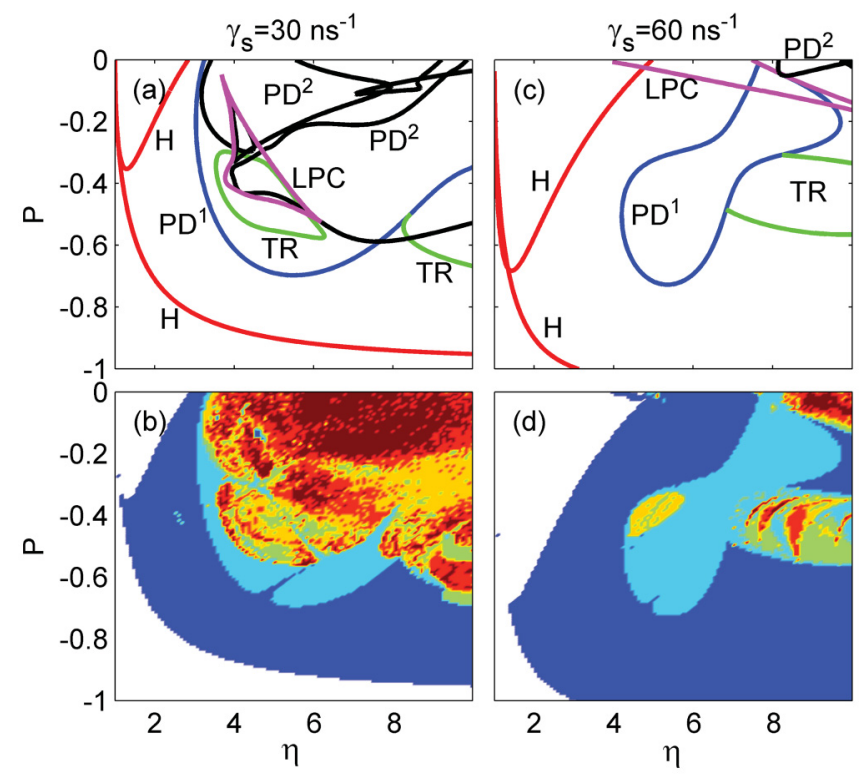

FIG. 11. (a),(c) The bifurcation diagrams and (b),(d) calculated color maps in the ( $\eta, P$ ) plane. (a),(b) $\gamma_{s}=30 \mathrm{~ns}^{-1}$ and (c),(d) $60 \mathrm{~ns}^{-1}$. In (b) and (d), the cw, P1, P2, and complicated dynamics including chaos are marked in white, dark blue, light blue, and other colors, respectively. limit cycle of basic period, and only the bifurcations forming the backbone of the dynamics are shown to keep the figure simple. Again, we stress that the two Hopf bifurcations are subcritical when they almost overlap due to the existence of the codimension- 2 generalized Hopf point, as mentioned in Fig. 6. When crossing the map either along $\eta$ or along $P$, one can find one-parameter bifurcations consistent with those obtained from direct time integration in Figs. 7 and 9. Moreover, the codimension-1 bifurcation curves in the diagram separate the parameter space into several distinct regions, which can be directly compared with the colored dynamical regions obtained from simulating Eqs. (1)-(4) [see Fig. 11(b)]. Here, the different dynamical regimes are identified from the extrema of the RCP intensity time series [43], in which cw, $\mathrm{P} 1, \mathrm{P} 2$, and complex dynamics are identified as a constant intensity, two intensity extrema, four intensity extrema, and even more extrema, respectively. The cw, P1, and P2 are marked in white, dark blue, and light blue, respectively. We use other colors to represent complicated dynamics including chaos where the number of extrema exceeds 4 and gradually increases using green to yellow, red, and dark red. It is clear that the bifurcation diagram in Fig. 11(a) is in good agreement with numerical studies in Fig. 11(b), but reveals much more detail, so that various bifurcation routes to chaos can be explained explicitly.

When a larger spin-flip relaxation rate is considered, i.e., $\gamma_{s}=60 \mathrm{~ns}^{-1}$, the bifurcation diagram shown in Fig. 11(c) is qualitatively different from the previous case. We see that the regions of complex dynamics and chaos shrink remarkably, e.g., only a closed $\mathrm{PD}^{1}$ bifurcation curve and a small segment of $\mathrm{PD}^{2}$ bifurcation curve outside $\mathrm{PD}^{1}$ are found. Comparing this with the colored bifurcation map in Fig. 11(d), one can conclude that the spin-flip relaxation rate strongly influences the size of regions of complex dynamics emerging from the bifurcations. This is expected since small values of $\gamma_{s}$ are beneficial for oscillations and complicated dynamics to occur. Again, based on our bifurcation analysis, extracting some physical implications behind the different dynamical regions becomes possible.

In Fig. 11, the difference between supercritical and subcritical bifurcations is not indicated since we provide the continuation bifurcation diagrams and those from the direct time integration of the SFM at the same time. One can easily have an idea about the stability of each bifurcation branch through comparing these two types of bifurcation maps. Indeed and as expected, one sees good agreement between them, and since most of the bifurcation curves shown are supercritical, distinct changes happen when crossing the bifurcation boundaries defined by the supercritical bifurcation curves. However, the presence of bi- and multistability behavior may lead to ambiguities. For instance, like any other laser systems, our spin VCSEL may exhibit the hysteresis phenomenon which indicates bistability between two different attractors (different basins of attraction) for the same set of bifurcation parameters $\eta$ and $P$. To address this, extensive simulations using a large number of initial conditions is one approach to reducing the discrepancies due to bi- and multistability. Alternatively, sweeping the bifurcation parameter both forward and backward can also be used to clarify the nature of the bistability. In Fig. 12, we show several one-parameter bifurcation diagrams 


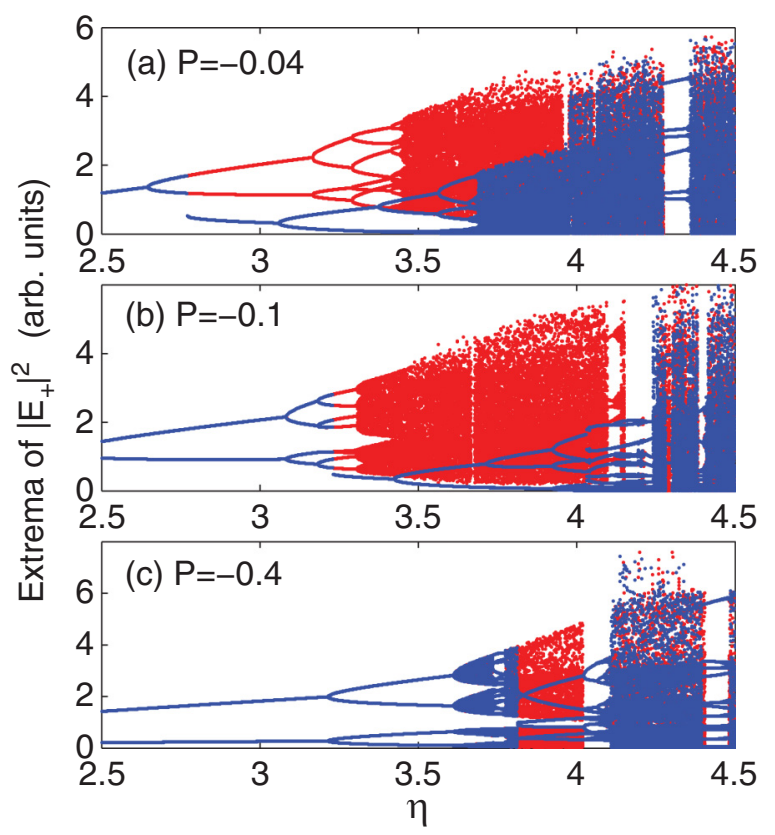

FIG. 12. Bifurcation diagrams of the RCP intensity as a function of $\eta$ for (a) $P=-0.04$, (b) -0.1 , and (c) -0.4 . The spin-flip relaxation rate $\gamma_{s}=30 \mathrm{~ns}^{-1}$. Other parameters are the same as those in Fig. 7. The red part is obtained for increasing $\eta$ and the blue part is obtained for decreasing $\eta$.

for three values of $P$ and for both increasing (red) and decreasing (blue) $\eta$. A forward and backward scan of the bifurcation parameter reveals that the hysteresis behavior remains for a finite interval of $\eta$, especially for $\eta<4.5$. Interestingly, from the bifurcation diagrams shown in Fig. 12, we encounter regions where there is a coexistence of different attractors, including cw with $\mathrm{P} 1, \mathrm{cw}$ with chaos, $\mathrm{P} 1$ with $\mathrm{P} 2, \mathrm{P} 1$ with higher periodic oscillations, $\mathrm{P} 1$ with chaos, $\mathrm{P} 2$ with chaos, and so on. A comparison among Figs. 12(a)-12(c) suggests that the width of the hysteresis cycle can be changed with varying $P$, and can even disappear, with the forward sweep being the same as the backward one for large $|P|$, roughly $|P|>0.4$. As a matter of fact, hysteresis occurs when we sweep $P$ back and forth, and keep $\eta$ constant. An example of this case is shown in Fig. 13. A comprehensive interpretation of this hysteresis behavior requires some additional bifurcation curves that are necessary to understand the occurrence of optical switching between different outputs of the spin VCSEL and is left outside the scope of the present study [31,32]; obviously, however, it can be associated with the supercritical LPC curves since when crossing these curves new limit cycle solutions can appear or disappear.

Finally, we argue that in the solitary spin VCSEL, there are many other bifurcation curves and some interesting codimension- 2 bifurcation points that are not indicated in the two-parameter bifurcation diagrams. A full description of bifurcations is beyond the scope of this paper, but we will discuss in some detail the LPC since some of them may be responsible for hysteresis. In Fig. 11(a), only one LPC curve is shown to keep the figure simple and give the reader a basic idea of the possible bifurcations. However, in Fig. 11(c), two LPC curves are given since a portion of them is supercritical, which

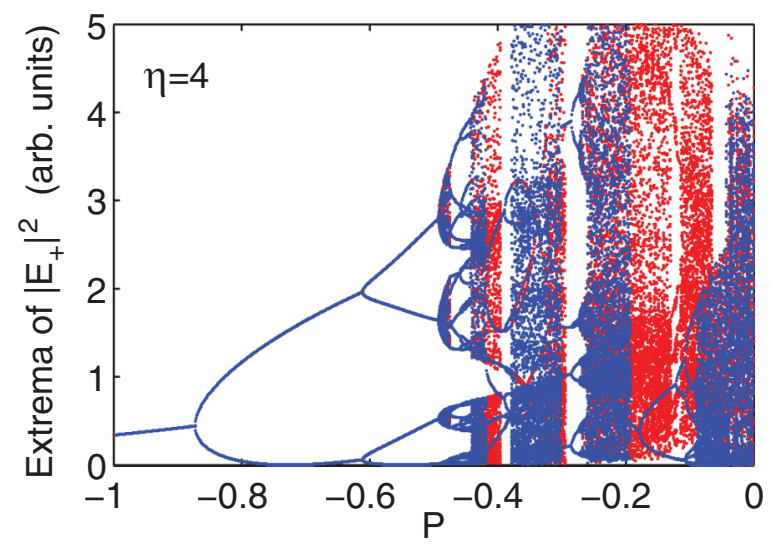

FIG. 13. Bifurcation diagram of the RCP intensity as a function of $P$ for $\eta=4$. Other parameters are the same as those in Fig. 9. The red part is obtained for increasing $P$ and the blue part is obtained for decreasing $P$.

can be determined by comparing with Fig. 11(d). To help the reader further understand the dynamics and the bifurcations, we present some more one-parameter bifurcation diagrams corresponding to the situation shown in Figs. 11(c) and 11(d), i.e., $\gamma_{s}=60 \mathrm{~ns}^{-1}$. Figure 14 shows bifurcation diagrams as a function of the pump ellipticity $P$ at three levels of the pump power $\eta$ : (a) 4.12, (b) 6, and (c) 10. In Fig. 14(a), one can notice a LPC around $P=-0.01$ [and a Hopf bifurcation at $P=-0.019$ that is not shown in Fig. 11(c)], which is supercritical, so Fig. 11(d) shows a narrow region of dark blue color corresponding to stable periodic orbit close to the top edge of the panel. In Fig. 14(b), no LPC is identified, so the limit-cycle oscillations remain when crossing the LPC

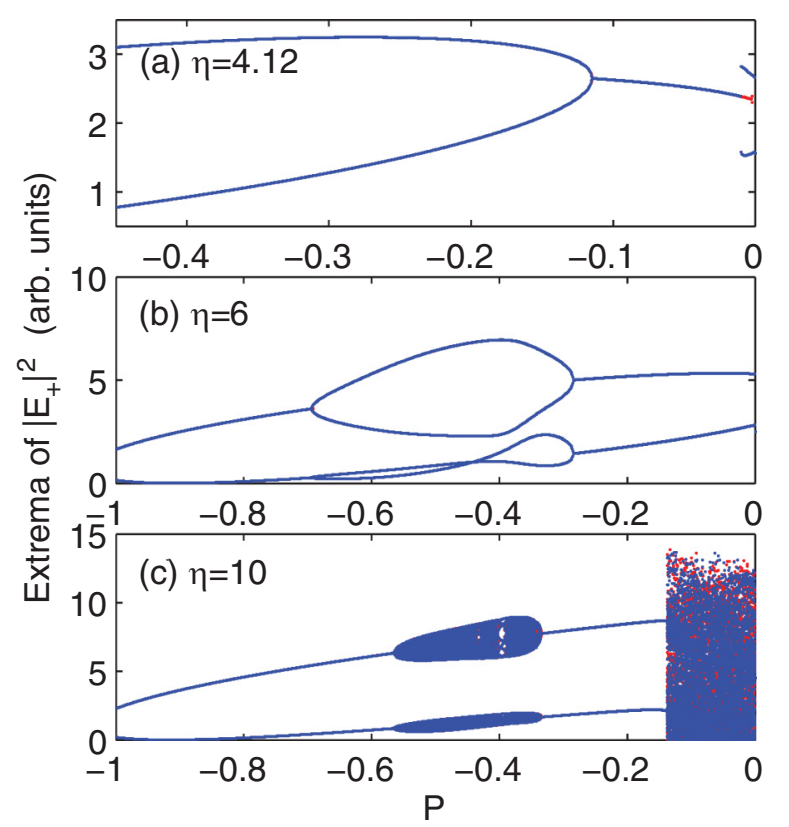

FIG. 14. Bifurcation diagrams of the RCP intensity as a function of $P$ for (a) $\eta=4.12$, (b) 6 , and (c) 10 . The spin-flip relaxation rate $\gamma_{s}=60 \mathrm{~ns}^{-1}$. Other parameters are the same as those in Fig. 9. The red part is obtained for increasing $P$ and the blue part is obtained for decreasing $P$. 
curve identified in Fig. 11(c), which implies that the LPC is subcritical. In Fig. 14(c), we encounter one supercritical LPC around $P=-0.14$ [the other one in Fig. 11(c) is unstable] where a transition to full-scale chaos occurs. The results of our combined AUTO-simulation (direct integration) study of bifurcations show a clear and simple way to determine the stability of bifurcation curves and interpret the dynamics. We stress here that there is no hysteresis in these three situations: computing the bifurcation by sweeping $P$ back and forth gives the same result.

\section{CONCLUSION}

In conclusion, we have carried out a detailed stability and bifurcation analysis of spin-polarized VCSELs. The importance of this work lies in two aspects. On the one hand, we extend and improve previous studies in [18], where the expansion was rather cumbersome and was carried out with respect to two small parameters representing the ellipticity and the difference between the total pump power and the lasing threshold. The previous results were acceptable only when the laser is biased extremely close to the threshold $(\eta \leqslant 1.0004)$ and for very small pump polarization $(P \ll 1)$. By contrast, here we perform asymptotic analysis by selecting a different scaling method and parameter. This produces more accurate approximations that are valid for larger ranges of pump magnitude $(\eta \leqslant 1.5)$ and for the whole interval of pump polarization ellipticity $(|P| \leqslant 1)$. It provides an alternative way to study the steady-state solutions, showing the possibility of identifying the stability of the equilibrium and predicting the switching behavior between different equilibria. However, we would like to point out that the present scalings only deal with the linear birefringence rate, the optical field decay rate, and the spin-flip relaxation rate due to the fact that they are usually much larger than other variables of the model. A more careful multiple-scale asymptotic expansion should be addressed in future work [44]. In addition, we provide detailed and systematic analysis of the nature of the bifurcations and dynamical regimes that can be accessed by a continuous tuning of the operating parameters. For this reason, we introduce reduced coordinate equations of the well-known spin-flip model by performing a transformation of variables for facilitating the usage of the standard numerical continuation software AUTO and, more importantly, identify physical implications of the dynamical regimes found in the spin VCSEL with the combination of direct time integration of the rate equations and the continuation technique. Various bifurcation scenarios, including a period-doubling and a quasiperiodic route to chaos, and hysteresis behavior are revealed.

\section{ACKNOWLEDGMENTS}

This work was supported by the Engineering and Physical Sciences Research Council (Grant No. EP/M024237/1). N.L. would like to thank Professor J. Sieber (University of Exeter) and Dr. M. Virte (Vrije Universiteit Brussels) for fruitful discussions of the continuation technique.

\section{APPENDIX A: COEFFICIENT MATRIX $\mathcal{M}$}

For convenience, the coefficient matrix $\mathcal{M}$ of the eigenvalue problem (7) is given as follows:

$$
\mathcal{M}=\left(\begin{array}{cccccc}
M_{11} & M_{12} & 0 & 0 & K_{1} E_{+} & K_{1} E_{+} \\
M_{12} & M_{22} & 0 & 0 & K_{1} E_{-} e^{i \theta} & -K_{1} E_{-} e^{i \theta} \\
0 & 0 & M_{11}^{*} & M_{12}^{*} & K_{1}^{*} E_{+} & K_{1}^{*} E_{+} \\
0 & 0 & M_{12}^{*} & M_{22}^{*} & K_{1}^{*} E_{-} e^{-i \theta} & -K_{1}^{*} E_{-} e^{-i \theta} \\
K_{2} E_{+} & K_{3} E_{-} e^{-i \theta} & K_{2} E_{+} & K_{3} E_{-} e^{i \theta} & M_{55} & M_{56} \\
K_{2} E_{+} & -K_{3} E_{-} e^{-i \theta} & K_{2} E_{+} & -K_{3} E_{-} e^{i \theta} & M_{56} & M_{66}
\end{array}\right),
$$

with

$$
\begin{aligned}
M_{11} & =\kappa\left(N_{s}+m_{s}-1\right)(1+i \alpha)-i \omega, \quad M_{12}=-\left(\gamma_{a}+i \gamma_{p}\right), \\
M_{22} & =\kappa\left(N_{s}-m_{s}-1\right)(1+i \alpha)-i \omega, \\
M_{55} & =-\gamma\left(1+E_{+}^{2}+E_{-}^{2}\right), \quad M_{56}=-\gamma\left(E_{+}^{2}-E_{-}^{2}\right), \quad M_{66}=-\left[\gamma_{s}+\gamma\left(E_{+}^{2}+E_{-}^{2}\right)\right], \\
K_{1} & =\kappa(1+i \alpha), \quad K_{2}=-\gamma\left(N_{s}+m_{s}\right), \quad K_{3}=\gamma\left(-N_{s}+m_{s}\right) .
\end{aligned}
$$

It is clear that the solution is unstable when there is an eigenvalue with $\operatorname{Re}(\lambda)>0$ and stable when all eigenvalues have $\operatorname{Re}(\lambda)<0$.

\section{APPENDIX B: VARIABLE TRANSFORMATION}

We begin with the complex conjugates of the two field equations (1) and (2) from the SFM model,

$$
\begin{aligned}
& \frac{d \bar{E}_{+}^{*}}{d t}=\kappa(N+m-1)(1-i \alpha) \bar{E}_{+}^{*}-\left(\gamma_{a}-i \gamma_{p}\right) \bar{E}_{-}^{*}, \\
& \frac{d \bar{E}_{-}^{*}}{d t}=\kappa(N-m-1)(1-i \alpha) \bar{E}_{-}^{*}-\left(\gamma_{a}-i \gamma_{p}\right) \bar{E}_{+}^{*} .
\end{aligned}
$$


We then obtain the following equations by taking complex conjugates and multiplying by the appropriate field or its complex conjugate:

$$
\begin{aligned}
& \bar{E}_{+} \frac{d \bar{E}_{+}^{*}}{d t}=\kappa(N+m-1)(1-i \alpha) \bar{E}_{+}^{*} \bar{E}_{+}-\left(\gamma_{a}-i \gamma_{p}\right) \bar{E}_{-}^{*} \bar{E}_{+}, \\
& \bar{E}_{-} \frac{d \bar{E}_{-}^{*}}{d t}=\kappa(N-m-1)(1-i \alpha) \bar{E}_{-}^{*} \bar{E}_{-}-\left(\gamma_{a}-i \gamma_{p}\right) \bar{E}_{+}^{*} \bar{E}_{-}, \\
& \bar{E}_{+}^{*} \frac{d \bar{E}_{+}}{d t}=\kappa(N+m-1)(1+i \alpha) \bar{E}_{+} \bar{E}_{+}^{*}-\left(\gamma_{a}+i \gamma_{p}\right) \bar{E}_{-} \bar{E}_{+}^{*}, \\
& \bar{E}_{-}^{*} \frac{d \bar{E}_{-}}{d t}=\kappa(N-m-1)(1+i \alpha) \bar{E}_{-} \bar{E}_{-}^{*}-\left(\gamma_{a}+i \gamma_{p}\right) \bar{E}_{+} \bar{E}_{-}^{*}, \\
& \bar{E}_{+}^{*} \frac{d \bar{E}_{-}}{d t}=\kappa(N-m-1)(1+i \alpha) \bar{E}_{-} \bar{E}_{+}^{*}-\left(\gamma_{a}+i \gamma_{p}\right) \bar{E}_{+} \bar{E}_{+}^{*}, \\
& \bar{E}_{-} \frac{d \bar{E}_{+}^{*}}{d t}=\kappa(N+m-1)(1-i \alpha) \bar{E}_{+}^{*} \bar{E}_{-}-\left(\gamma_{a}-i \gamma_{p}\right) \bar{E}_{-}^{*} \bar{E}_{-} .
\end{aligned}
$$

The variable $\Omega$ is defined as

$$
\Omega=\left|\bar{E}_{+}\right|^{2}-\left|\bar{E}_{-}\right|^{2}=\bar{E}_{+} \bar{E}_{+}^{*}-\bar{E}_{-} \bar{E}_{-}^{*},
$$

which is real valued and, by taking the first derivative, we obtain

$$
\frac{d \Omega}{d t}=\frac{d \bar{E}_{+} \bar{E}_{+}^{*}}{d t}-\frac{d \bar{E}_{-} \bar{E}_{-}^{*}}{d t}
$$

where

$$
\frac{d \bar{E}_{+} \bar{E}_{+}^{*}}{d t}=\bar{E}_{+} \frac{d \bar{E}_{+}^{*}}{d t}+\bar{E}_{+}^{*} \frac{d \bar{E}_{+}}{d t}=2 \kappa(N+m-1)\left|\bar{E}_{+}\right|^{2}-\gamma_{a}\left(\bar{E}_{-}^{*} \bar{E}_{+}+\bar{E}_{-} \bar{E}_{+}^{*}\right)+i \gamma_{p}\left(\bar{E}_{-}^{*} \bar{E}_{+}-\bar{E}_{-} \bar{E}_{+}^{*}\right)
$$

and

$$
\frac{d \bar{E}_{-} \bar{E}_{-}^{*}}{d t}=\bar{E}_{-} \frac{d \bar{E}_{-}^{*}}{d t}+\bar{E}_{-}^{*} \frac{d \bar{E}_{-}}{d t}=2 \kappa(N-m-1)\left|\bar{E}_{-}\right|^{2}-\gamma_{a}\left(\bar{E}_{+}^{*} \bar{E}_{-}+\bar{E}_{+} \bar{E}_{-}^{*}\right)+i \gamma_{p}\left(\bar{E}_{+}^{*} \bar{E}_{-}-\bar{E}_{+} \bar{E}_{-}^{*}\right) .
$$

Substituting Eqs. (B11) and (B12) into Eq. (B10), we arrive at

$$
\frac{d \Omega}{d t}=2 \kappa(N-1) \Omega+2 \kappa m\left(\left|\bar{E}_{+}\right|^{2}+\left|\bar{E}_{-}\right|^{2}\right)+2 i \gamma_{p}\left(\bar{E}_{-}^{*} \bar{E}_{+}-\bar{E}_{-} \bar{E}_{+}^{*}\right) .
$$

Similarly, we define another variable $Q=\bar{E}_{-} \bar{E}_{+}^{*}$ and write its first derivative as

$$
\frac{d Q}{d t}=\bar{E}_{-} \frac{d \bar{E}_{+}^{*}}{d t}+\bar{E}_{+}^{*} \frac{d \bar{E}_{-}}{d t}=2 \kappa Q(N-1-i \alpha m)-\gamma_{a}\left(\left|\bar{E}_{+}\right|^{2}+\left|\bar{E}_{-}\right|^{2}\right)-i \gamma_{p}\left(\left|\bar{E}_{+}\right|^{2}-\left|\bar{E}_{-}\right|^{2}\right) .
$$

Taking into account all the previous transformations, the SFM becomes

$$
\begin{gathered}
\frac{d \Omega}{d t}=2 \kappa(N-1) \Omega+2 \kappa m \sqrt{\Omega^{2}+4|Q|^{2}}+i 2 \gamma_{p}\left(Q^{*}-Q\right), \\
\frac{d Q}{d t}=2 \kappa Q(N-1-i \alpha m)-\gamma_{a} \sqrt{\Omega^{2}+4|Q|^{2}}-i \gamma_{p} \Omega, \\
\frac{d N}{d t}=\gamma\left[\eta-\left(1+\sqrt{\Omega^{2}+4|Q|^{2}}\right) N-\Omega m\right], \\
\frac{d m}{d t}=\gamma P \eta-\left(\gamma_{s}+\gamma \sqrt{\Omega^{2}+4|Q|^{2}}\right) m-\gamma \Omega N .
\end{gathered}
$$

It is obvious that $Q$ is the only complex-valued variable and can be rewritten in terms of real and imaginary parts, so we get Eqs. (27)-(31) which have been used for continuation results.

[1] S. Datta and B. Das, Appl. Phys. Lett. 56, 665 (1990).

[2] J. Sinova and I. Žutić, Nat. Mater. 11, 368 (2012).
[3] N. C. Gerhardt and M. R. Hofmann, Adv. Opt. Technol. 2012, 268949 (2012). 
[4] I. Žutić, J. Fabian, and S. D. Sarma, Rev. Mod. Phys. 76, 323 (2004).

[5] S. Hövel, N. Gerhardt, M. Hofmann, J. Yang, D. Reuter, and A. Wieck, Electron. Lett. 41, 251 (2005).

[6] S. Hövel, A. Bischoff, N. C. Gerhardt, M. R. Hofmann, T. Ackemann, A. Kroner, and R. Michalzik, Appl. Phys. Lett. 92, 041118 (2008).

[7] M. J. Adams and D. Alexandropoulos, IEEE J. Quantum Electron. 45, 744 (2009).

[8] D. Saha, D. Basu, and P. Bhattacharya, Phys. Rev. B 82, 205309 (2010).

[9] P. E. F. Junior, G. Xu, J. Lee, N. C. Gerhardt, G. M. Sipahi, and I. Žutić, Phys. Rev. B 92, 075311 (2015).

[10] M. Holub, J. Shin, D. Saha, and P. Bhattacharya, Phys. Rev. Lett. 98, 146603 (2007).

[11] M. Holub and P. Bhattacharya, J. Phys. D: Appl. Phys. 40, R179 (2007).

[12] K. Ikeda, T. Fujimoto, H. Fujino, T. Katayama, S. Koh, and H. Kawaguchi, IEEE Photon. Technol. Lett. 21, 1350 (2009).

[13] S. S. Alharthi, A. Hurtado, R. K. Al Seyab, V.-M. Korpijärvi, M. Guina, I. D. Henning, and M. J. Adams, Appl. Phys. Lett. 105, 181106 (2014).

[14] S. S. Alharthi, J. Orchard, E. Clarke, I. D. Henning, and M. J. Adams, Appl. Phys. Lett. 107, 151109 (2015).

[15] J. Rudolph, D. Hägele, H. M. Gibbs, G. Khitrova, and M. Oestreich, Appl. Phys. Lett. 82, 4516 (2003).

[16] D. Basu, D. Saha, and P. Bhattacharya, Phys. Rev. Lett. 102, 093904 (2009).

[17] K. Schires, R. K. Al Seyab, A. Hurtado, V.-M. Korpijärvi, M. Guina, I. D. Henning, and M. J. Adams, Opt. Express 20, 3550 (2012).

[18] H. Susanto, K. Schires, M. J. Adams, and I. D. Henning, Phys. Rev. A 92, 063838 (2015).

[19] N. Li, D. Alexandropoulos, I. D. Henning, and M. J. Adams, Electronics 5, 83 (2016).

[20] M. Virte, K. Panajotov, H. Thienpont, and M. Sciamanna, Nat. Photon. 7, 60 (2013).

[21] M. Virte, K. Panajotov, and M. Sciamanna, Phys. Rev. A 87, 013834 (2013).

[22] F. Prati, P. Caccia, M. Bache, and F. Castelli, Phys. Rev. A 69, 033810 (2004).

[23] A. Gahl, S. Balle, and M. San Miguel, IEEE J. Quantum Electron. 35, 342 (1999).
[24] N. C. Gerhardt, M. L. Li, H. Jähme, H. Höpfner, T. Ackemann, and M. R. Hofmann, Appl. Phys. Lett. 99, 151107 (2011).

[25] M. Lindemann, T. Pusch, R. Michalzik, N. C. Gerhardt, and M. R. Hofmann, Appl. Phys. Lett. 108, 042404 (2016).

[26] K. Schires, R. K. Al Seyab, A. Hurtado, V. M. Korpijärvi, M. Guina, I. D. Henning, and M. J. Adams, in IEEE Photonics Conference (IPC), September 23-27, 2012, San Francisco (IEEE, Piscataway, NJ, 2012), pp. 870-871.

[27] S. S. Alharthi, R. K. Al Seyab, I. D. Henning, and M. J. Adams, IET Optoelectron. 8, 117 (2014).

[28] R. K. Al Seyab, D. Alexandropoulos, I. D. Henning, and M. J. Adams, IEEE Photon. J. 3, 799 (2011).

[29] S. Y. Xiang, W. Pan, B. Luo, L. S. Yan, X. H. Zou, N. Jiang, L. Yang, and H. N. Zhu, J. Lightwave Technol. 29, 2173 (2011).

[30] J. Martin-Regalado, F. Prati, M. San Miguel, and N. B. Abraham, IEEE J. Quantum Electron. 33, 765 (1997).

[31] W. L. Zhang, W. Pan, B. Luo, M. Y. Wang, and X. H. Zou, IEEE J. Sel. Top. Quantum Electron. 14, 889 (2008).

[32] J. Paul, C. Masoller, P. Mandel, Y. Hong, P. S. Spencer, and K. A. Shore, Phys. Rev. A 77, 043803 (2008).

[33] J. Mulet, C. R. Mirasso, and M. San Miguel, Phys. Rev. A 64, 023817 (2001).

[34] M. S. Torre, H. Susanto, N. Li, K. Schires, M. F. Salvide, I. D. Henning, M. J. Adams, and A. Hurtado, Opt. Lett. 42, 1628 (2017).

[35] E. J. Doedel, A. R. Champneys, T. Fairgrieve, Y. Kuznetsov, B. Oldeman, R. Pfaffenroth, B. Sandastede, X. Wang, and C. Zhang, AUTO-07p: Continuation and Bifurcation Software for Ordinary Differential Equations (Concordia University, Montreal, 2008).

[36] V. Crockett, Ph.D. thesis, University of Exeter, 2010.

[37] J. Sieber, SIAM J. Appl. Dyn. Syst. 1, 248 (2002).

[38] K. E. Chlouverakis and M. J. Adams, Opt. Commun. 216, 405 (2003).

[39] T. Fordell, A. M. Lindberg, Opt. Commun. 242, 613 (2004).

[40] C. Bonatto and J. A. C. Gallas, Phys. Rev. E 75, 055204(R) (2007).

[41] B. Krauskopf, S. Wieczorek, and D. Lenstra, Appl. Phys. Lett. 77, 1611 (2000).

[42] J. Mørk, B. Tromborg, and J. Mark, IEEE J. Quantum Electron. 28, 93 (1992).

[43] M. Sciamanna and K. Panajotov, Phys. Rev. A 73, 023811 (2006).

[44] L. Gil and G. L. Lippi, Phys. Rev. Lett. 113, 213902 (2014). 